J Immigr Minor Health. 2018 April ; 20(2): 283-294. doi:10.1007/s10903-017-0565-9.

\title{
SEX DIFFERENCES AND PREDICTORS OF CHANGES IN BODY WEIGHT AND NONCOMMUNICABLE DISEASES IN A RANDOM, NEWLY-ARRIVED GROUP OF REFUGEES FOLLOWED FOR TWO YEARS
}

\author{
K-L. CATHERINE JEN, PH.D. ${ }^{1}$, HIKMET JAMIL, MD, PH.D. ${ }^{2,3}$, KEQUAN ZHOU, PH.D. ${ }^{1}$, \\ KAREN BREEJEN, B.A. ${ }^{2}$, and BENGT B. ARNET, MD, PH.D. ${ }^{2,3,4}$ \\ ${ }^{1}$ Department of Nutrition and Food Science, Wayne State University \\ ${ }^{2}$ Department of Family Medicine and Public Health Sciences, Wayne State University \\ ${ }^{3}$ Department of Family Medicine, College of Human Medicine, Michigan State University \\ ${ }^{4}$ Department of Public Health and Caring Sciences, Uppsala University, Uppsala, Sweden
}

\section{Abstract}

Background-We have reported that none of the psychological/mental variables examined predicted the increase in BMI and non-communicable diseases (NCDs) in Iraqi refugees after one year resettlement in Michigan.

Methods-We continuously followed the same cohort of refugees for 2 years (Y2 FU) to further determine the gender difference in predicting of increased BMI and NCDs.

\begin{abstract}
Results-Only $20 \%$ of the BMI variability could be accounted for by the factors examined. Number of dependent children and depression were positively and stress negatively associated with BMI in male refugees but not in females. Number of dependent children was negatively associated with changes in BMI and in males only. Two third of the NCD variability was accounted for by gender, BMI, employment status, depression, posttraumatic stress disorders and coping skills. Unmarried, unemployed and with high PTSD scores at Y2 in males were positively and number of dependent children was negatively associated with NCD changes in females.
\end{abstract}

Discussion-Factors such as dietary patterns and lifestyle may have contributed to the increased in BMI and NCDs in these refugees at 2 years post-settlement.

\section{INTRODUCTION}

\begin{abstract}
Several studies have reported that Middle Eastern (ME) refugees have a high prevalence of mental or behavioral disorders after resettlement $[1,2]$. We have previously shown that Iraqi refugees suffer from more posttraumatic stress disorders (PTSD), trauma, depression, and other mental health issues than the general population and ME immigrants who have not
\end{abstract}

Corresponding author: Dr. K-L. Catherine Jen, Department of Nutrition and Food Science, Wayne State University, Detroit, MI 48202 , Phone: 313-577-1948; fax: 313-577-8616, cjen@wayne.edu.

No conflict of interest for all authors 
been exposed to war [3, 4]. However, work to date is mostly cross-sectional, retrospective or based on studies of non-random samples of refugees that have already resided in their new country for a period of time [5-7]. This makes it difficult to delineate whether chronic disease conditions express themselves before or after displacement from the old country and the relationship between risk factors and disease processes. In the study conducted by Dookeran et al [7], significant numbers of refugees and asylees suffered from overweight/ obesity and hypertension. However, the rates of heart disease, diabetes and anemia were low relative to the U.S. population. Our previous research has also shown that Iraqi refugees gained weight rapidly and had significant increases in hypertension, stress and depression at the end of year one FU (Y1 FU) [8]. It is known that psychological distress increases with length of stay [9]. Hence strategies to maintain refugees' mental and physical health after resettlement in the U.S. should be a top priority in public health.

In our previous study, we investigated a number of mental/psychological and physical health factors but none predicted increase in body mass index (BMI) at Y1 FU [8]. After resettlement in a new country, refugees not only suffer prior and ongoing stress or trauma, but may also experience financial strains, acculturation pressure and an undesirable neighborhood. Iraqi refugees are twice as likely to be unemployed than Iraqi immigrants [10], suggesting a more difficult economic condition for refugees. The economic conditions could affect diet pattern since individuals with low SES spend less money per $1000 \mathrm{kcal}$ of food and are more likely to purchase food high in fat but low in nutrient content [11]. This increases the risk for increased energy intake and obesity. Collectively, environmental factors that refugees commonly face could directly and indirectly contribute to the increased risk of obesity and NCDs [12], contributing to the health disparities in refugees.

We have reported no sex difference in BMI at the time Iraqi refugees arrived in the US. However, after one year in the US, female refugees had gained more weight than males. Linear regression analyses were not able to identify significant predictors for this sex-related difference [8]. The purpose of this study was to continue to track BMI and NCDs in our cohort of Iraqi refugees during Y2 FU and to determine what risk factors might be predictive of adverse weight and NCD development and to what degree there are sex-related differences.

\section{METHODS}

\section{Participants}

Detailed description of the refugee sample has been presented in a previous study depicting the changes during the first year post-resettlement [8]. In brief, 298 refugees were randomly selected from a computer generated list of newly arrived refugees provided by our community partners. For the FU, 290 refugees were still remaining at the end of Y1, and 282 at $\mathrm{Y} 2$. The retention rates are $97.3 \%$ and $94.6 \%$ for $\mathrm{Y} 1$ and $\mathrm{Y} 2$, respectively. The mean age at baseline level (BL) was 33.4 \pm 11.3 years old (18-69 years) and $137(46 \%)$ were females. The mean time that participants had been in the U.S. at the BL, Y1 and Y2 FU assessment was $1.0 \pm 1.1$ (mean \pm SD) months, $12.5 \pm 1.1$, and $24.8 \pm 1.5$ months respectively. Other description of these refugees are presented in Table 1. 
Informed consent was obtained from all participating refugees. All procedures and materials were approved by the Institutional Review Board of Wayne State University (Protocol\#: 025509B3F).

\section{Measurements}

All the questionnaires and measurements used in this study have been described previously and were administered in a structured interview procedure [8] (Table 2). All these measures were administered at BL, Y1 and Y2. Employment status was determined by a yes or no answer to a question: are you currently working? Persistent financial problems was the dichotomized answer (sometimes, often, almost always vs almost never and rarely) to the question: "How often do you experience daily money problems?" Post-displacement stressors was calculated as the sum of all "yes" responses to the Harvard Trauma Questionnaire questions.

\section{Statistical analysis}

A composite score of NCD was calculated as the sum (yes or no) of hypertension, CVD, hypercholesterolemia, diabetes, asthma and arthritis. Changes in BMI and NCDs were calculated as the differences between BL and Y2 FU values. Analysis of variance with repeated measures was used to analyze BMI and other variables over the 2 year period. A 2 $\times 2$ factorial analysis was used to determine the interaction of sex and marital and employment status, number of dependent children and financial problems at Y2 on BMI, $\mathrm{NCD}$ and their changes.

Since the residues of BMI, BMI changes, NCDs and NCD changes were normally distributed, a 4-model linear regression was conducted to identify potential predictors of, or factors associated with BMI or changes in BMI at Y2 and NCD or NCD changes at $\mathrm{Y} 2$ using data collected both at BL and Y2. In model 1, BMI and NCDs were predicted by age, sex, education levels, marital status, number of dependent children, and employment status. In model 2, stress levels, PTSD, depression and discrimination at BL or Y2 were entered as potential predictors or associated factors. Financial strain and post-displacement stressors were added in model 3. In model 4, scores of social support, resilience, life-meaning, coping and acculturation were added. All statistical analyses were performed using IBM SPSS version 23 (IBM Corp., Armonk, NY). The significance level was set to a two-tailed $\mathrm{p}<0.05$.

\section{RESULTS}

\section{Self-reported health}

Number of refugees who reported having "excellent health" decreased from $31.1 \%$ at BL to $12.4 \%$ at $\mathrm{Y} 2$ while reporting of "good health" increased from $12.0 \%$ to $36 \%$ during the same period of time ( $\mathrm{p}=0.003)$. Percentages of refugees who reported as having "very good", "fair" and "poor" health did not change. Similar changes in self-reported mental health status were observed over the 2 year period $(\mathrm{p}<0.0001)$. 


\section{$\mathrm{BMI} /$ changes in BMI}

The mean BMI at BL, Y1 and Y2 FU levels were 26.6 $\pm 4.9,26.9 \pm 4.8$ and $27.4 \pm 4.9 \mathrm{~kg} / \mathrm{m}^{2}$ respectively. There was a significant increase in BMI $(\mathrm{p}<0.0001)$ and an upward shift from lower categories to higher ones over time $(\mathrm{p}=.002$, Figure 1$)$.

Sex difference in BMI changes-Female refugees had significantly lower BMI at BL than males ( $25.7 \pm 4.6$ vs $27.2 \pm 5.0, \mathrm{p}=.007$ ) but gained significant more weight than males during the 2 year $\mathrm{FU}\left(1.4 \pm 0.2 \mathrm{~kg} / \mathrm{m}^{2}\right.$ for females and $0.4 \pm 0.2 \mathrm{~kg} / \mathrm{m}^{2}$ for males, $\left.\mathrm{p}=0.001\right)$. BMI at Y2 showed no sex difference. Number of dependent children at Y2 was positively correlated with BMI at Y2 FU for male but not with females. Changes in BMI and number of dependent children were correlated in both male and female refugees (Table 3).

There were sex associated differences in the relationships between BMI at $\mathrm{Y} 2$ and psychological/mental factors. BMI of female refugees were negatively correlated with discrimination, social support, resilience, life meaning, coping, and acculturation (Table 3). Females' BMI was positively correlated with stress, depression, PTSD, total NCDs, post displacement stressors and financial problems. For male refugees, BMI was not related to social support, resilience, life meaning, coping skills, acculturation, post-displacement stressors and financial problems. However, male refugees' BMI was positively correlated with depression, PTSD and total NCDs, and negatively correlated with discrimination. No sex difference in the relationships between changes in BMI and all the factors examined except BMI at Y2 FU and number of dependent children for both sexes.

\section{Effects of interaction of sex and other demographic factors at Y2 in BMI at Y2} and BMI changes-Married refugees had higher BMI than singles (divorced, widowed or separated) (Table 4). There was no interaction of sex and marital status on BMI or changes in BMI. Refugees with dependent children had significantly higher BMI than those without (Table 4). Females with children gained more weight than males with children. Hence a significant interaction of sex and with/without children was observed. Refugees who were employed at Y2 had lower BMI than unemployed. There was no sex by employment interaction on BMI, nor BMI changes.

Refugees with persistent financial problems had higher BMI at Y2 FU (p=.001). However, no difference in changes in BMI was observed between those with and without persistent financial problems. No interaction between financial problems and sex was observed for BMI or BMI changes.

\section{NON-COMMUNICABLE DISEASES AT Y2 FU}

There was a significant upward trend toward higher number of NCDs with increasing time in the US ( $p<.0001$, Table 5). No sex difference was observed neither in total number of NCDs, nor in the numbers of new NCDs during the first 2 years. Changes in NCD were related to different factors in males and females (Table 6).

\section{Effects of interaction of sex and other demographic factors at Y2 in NCDs at} Y2 and NCD changes-Married refugees had significantly higher number of NCDs as 
compared to singles (Table 4). There were significant interactions between sex and marital status at Y2 ( $\mathrm{p}<.0001)$ on total and changes in NCDs. The changes in NCD numbers according to sex and number of dependent children at $\mathrm{Y} 2$ also show the similar pattern. There were significant interactions between sex and employment status at Y2. Refugees experienced persistent financial problems at $\mathrm{Y} 2$ had higher number of NCDs and new NCDs than those without financial problems. No sex and financial problem interaction on NCD nor changes in NCD was observed.

\section{PREDICTING BMI, BMI CHANGES AND NCD AND THEIR CHANGES USING LINEAR REGRESSIONS BMI at Y2 FU}

A 4-model linear regression analysis revealed that BMI at Y2 could only be predicted positively in all models by BMI at BL, but negatively predicted by number of dependent children in model 4. No other BL data predicted BMI at Y2. On the other hand, using Y2 data, BMI at Y2 was positively associated with sex, age, number of dependent children, depression, and negatively by discriminations when both sexes were combined at Y2 (Table 7). Females' BMI was not associated with any of the variables studied at Y2 while males' BMI was positively associated with the number of dependent children, depression, and negatively by stress at Y2. Only $20 \%$ of the variation in BMI at Y2 FU could be accounted for by all the factors examined at Y2.

Changes in BMI from BL to Y2 FU-For all the BL data collected, BMI changes could be negatively predicted by BML at BL and number of dependent children. BMI changes were associated with following data collected at Y2: BMI, marital status (married gained more weight), and age (younger refugees gained more weight). For females, Y2 age negatively and Y2 stress positively associated with changes in BMI ( $p<.05)$. Married male refugees gained more weight but number of dependent children was negatively associated with the BMI changes. With all possible Y2 factors entered into the linear regression, only $12 \%$ of the variation in BMI changes can be predicted.

NCDs-Total number of NCDs at Y2 was positively predicted by age and BMI at BL but negatively by education levels at BL. Table 8 presented associations of different factors at Y2 and NCDs at Y2. Sex (males), age, BMI at Y2, unemployment, depression at Y2, PTSD at $\mathrm{Y} 2$ and poor coping skills were positively while number of dependent children was negatively associated with higher number of NCDs when both sexes were combined. Total NCDs was also associated with higher post-displacement stressors in model 4. Females with older age, higher BMI at Y2, and unemployed had more NCDs while those with more dependent children, and with healthy coping skills at Y2 had lower number of NCDs. Male sex, older age, higher PTSD and depression scores at Y2, and unemployment at Y2 were associated with a higher number of NCDs. About two thirds of the variation in NCD numbers could be explained by the factors examined.

Changes in NCDs-NCD changes from BL to Y2 were predicted by age and BMI at BL in model 1. In model 2, BL age, BMI and depression at BL were positive predictors for NCD changes while number of dependent children negatively predicted NCD changes. In 
models 3 and 4, BMI at BL lost its predicting power. Using data collected at Y2, it was identified that age, BMI and unemployment at $\mathrm{Y} 2 \mathrm{FU}$ were associated with more changes in NCDs. More dependent children was associated with lower number of new NCDs. Changes in females' NCD were associated positively with BMI at Y2 and negatively by number of dependent children. For males, being married, unemployed and having higher PTSD scores at Y2 indicated higher number of new NCDs. Only a quarter of the variation in NCD changes could be accounted for by the factors examined.

\section{DISCUSSION}

This study is unique in that we were able to follow a random sample of newly arrived refugees to determine predictors of changes in BMI and NCD load during their first 2 years in the US. We have previously reported a significant BMI increase and an upward shift in BMI distribution during refugees' first year in the US. Furthermore, the number of refugees diagnosed with hypertension increased during their first year [8]. Similar results have been reported by others [7, 13]. In this 2-Year FU study, body weight and number of new NCDs continued to increase. Sex differences were observed in factors associated with BMI and $\mathrm{NCD}$ at $\mathrm{Y} 2 \mathrm{FU}$.

\section{Factors associated with BMI and BMI changes at Y2 FU}

Contrary to the common belief, number of dependent children at Y2 was correlated with higher BMI at Y2 FU for males but not for females even though females with dependent children gained more weight than females without children. Changes in BMI in both males and females were positively correlated with number of dependent children. Yun et al has reported that refugees without children were less likely to become overweight, but equally likely to become obese compared with those with dependent children, although their study did not examine possible sex effects [13]. It has been reported that women's weight is positively related to parity but there are many modifiers (race, sociodemorgraphic, age) that influence this relationship $[14,15]$. There is no study investigating such relationship in fathers, hence it is not clear if this is a direct association or through yet unidentified factors that generate this result.

When examining changes in BMI from BL to Y2, having more dependent children was associated smaller changes in BMI in males but more changes in females. Factors other than those investigated in this study must have contributed to the weight gain in females and deserve further investigation.

Using Pearson's correlation analyses, our data revealed that for females, BMI at Y2 was positively correlated with many negative psychological variables, post-displacement stressors and financial problems but negatively correlated with social support, resilience, life meaning, coping skill and acculturation. No such relationship existed in males between factors associated with positive traits and BMI, suggesting a significant sex difference in responding to environmental factors. A positive attitude and a supportive environment may have alleviated some of the adverse effects of post-displacement stressors in trajectory of body weight in females even though females still gained more weight than males. However, linear regression analysis did not reveal any contributions of these factors to BMI at Y2. 
Apparently, these factors must have worked through a common underline mechanism to produce their effects on BMI at Y2. To identify the common pathway and its effect on body weight should be a future research goal.

Multiple regression analysis did not identify acculturation as a predictor of BMI or changes in BMI at Y2. However, acculturation was negatively correlated with BMI in females, with no association in males. Our findings do not agree with those reported by others. For example, in Nelson-Peterman et al's cross-sectional study, female Cambodian refugees with higher acculturation scores had elevated BMI [16]. For Latinos, acculturation also contributes to higher BMI $[17,18]$. Delavari et al reviewed the literature and identified eight cross-sectional and one longitudinal (one year) studies investigating the effect of acculturation on body weight among migrant population residing in affluent countries [19]. Positive correlations were reported in six studies. The remaining three reported negative relationships in females. It is apparent that sex interacts with acculturation to affect BMI. Acculturated females in affluent countries may have adopted the culture of host countries and practiced dieting behavior to prevent weight gain. This may also partially explain why there was no relationship between number of dependent children and BMI, and there is no factor other than age that predicted BMI or BMI change for females. On the other hand, the length of stay in the host country was positively correlated with BMI [19]. It should be noted that the current study followed a random sample of newly arrived refugees for two years with very low attrition rate, while most of previously research used cross-sectional samples or with only one year FU. Therefore, any differences between baseline and Y2 FU observed in the current study represent the real changes within each refugee after resettlement. This longitudinal design provides more accurate description regarding refugees' postdisplacement changes in mental and physical well-being and identifies new stressors they face in the host countries.

Stress affects body weight in a complex fashion. Acute stress may reduce food intake hence body weight while chronic stress may increase food intake and weight gain, especially in men [20]. In our study, we observed that using regression analysis, stress was negatively associated with BMI at Y2 in males but not in females. However, stress at Y2 positively predicted BMI changes in females but not in males. Matheson et al have reported that men residing in poor neighborhoods had higher stress levels and gained less weight while women gained more weight than those living in affluent neighborhoods [21]. It is known that when under stress, women are more likely using food to manage stress while men are more likely using smoking or drinking as a way of dealing with stress [22]. We did observe a significant increase in drinking and smoking cigarette/hookah behaviors (data not shown) in males. This sex difference in behavior may explain the difference in predicting BMI and BMI changes. Therefore, future intervention strategies should be sex specific and take neighborhood conditions into consideration as suggest by Matheson et al [21].

Even with all the factors investigated in this study, only about $20 \%$ of the variation in BMI at Y2 and 12\% in BMI changes could be explained. Hence factors not included in this study accounted for the majority of the variation in BMI. It is speculated that eating behavior/food intake patterns and lifestyle may contribute significantly to these variations and deserve further investigation. 


\section{Predictors for NCDs and NCD changes}

Our data showed that BMI and NCD were regulated by different factors. NCDs are more likely related to the psychological/mental factors and employment status while BMI relates to other factors in need of detailed studies in the future. Similarly, about $25 \%$ of the changes in the numbers of NCDs can be accounted for. There is an urgent need to identify these "other factors" which contribute to the changes during the early post-displacement years.

When the refugees arrived in the U.S., their NCD rates were lower than the general U.S. adult population, suggesting that upon arrival in the US, refugee's health status was generally better than that of U.S. adults, which has been referred to as the "healthy refugee" effect [23]. Significant increases in percentages of refugees suffering from hypertension, diabetes, hypercholesterolemia and arthritis were observed over the 2-year FU. The prevalence of diabetes at Y2 FU was not different from the general U.S. population. As refugee settle in a new country and start to acculturate to the new culture and environment, their health status decline [9, 23]. Early post-settlement period becomes a critical period to establish a healthy lifestyle and eating habits for refugees. If no effective intervention is in place during the early period, rates of NCD in refugees will catch up with those of the U.S. adult population.

With linear regression analysis, post-displacement stressors and persistent financial problems were not associated BMI, BMI changes, total NCD at Y2 and NCD changes. However, employment status and financial problems were correlated to BMI and NCDs at Y2. Employment status exerted more influence in changes in BMI and NCDs than persistent financial problems. It is postulated that employment, regardless of the level of employment, may have alleviated some the financial burdens and thus reduced stressors and the resulted changes in NCDs.

From the linear regression results of BMI and NCDs, it can be concluded that psychological/ mental factors affects total NCDs at Y2 but not NCD changes, nor BMI/BMI changes. These results point to a need to identify other factors not examined in this study as predictors of weight gain and NCDs, including diet and physical activity patterns.

\section{Limitations}

Lack of records of diet and daily activity levels is a limitation since only a small portion of the variations in BMI, BMI changes and NCD changes can be predicted by factors examined in this study. Furthermore, a majority of refugees in this study were Christians, the results may not apply to other religious groups from ME region. This study only followed the refugees for 2 years, while some of the NCDs take a longer period to develop. Future studies should recruit newly arrived ME refugees from various religion groups, follow them up for several years and include even more comprehensive data, including dietary and daily physical activity records.

\section{Conclusion}

By being able to prospectively follow a random sample of newly arrived refugees, we were able to measurably enhance our understanding of risk factors for adverse health development 
in males. However, our model was less successful in predicting adverse health changes in females. Future research needs more details to determine changes in exposure to risk and resilience factors in male vs female refugees, as well as possible changes in biopsychosocial reactions to such stressors and resilience factors.

\section{Acknowledgments}

\section{Compliance with Ethical Standards}

This study was supported by a grant from the National Institute of Mental Health (NIMH, R01MH085793). Drs. Arnetz and Jamil are also partially supported by a grant from the National Institute of Environmental Health Sciences (P30ES020957). We would like to thank our community partners and all refugees that volunteered to take part in this study. Without their strong support and commitment, the study would not have been possible. The content is solely the responsibility of the authors and does not necessarily reflect the official view of NIMH. All authors declare that there is no conflict of interest. All procedures performed in this study were in accordance with the ethical standards of the institutional research committee and with the 1964 Helsinki declaration and its later amendments or comparable ethical standards. Informed consent was obtained from all refugees participating in this study.

\section{References}

1. Llosa A, Ghantous Z, Souza R, Forgione F, Bastin P, Jones A, et al. Mental disorders, disability and treatment gap in a protracted refugee setting. Br J Psychiatry. 2014; 204:208-13. [PubMed: 24029537]

2. Taylor E, Yanni E, Pezzi C, Guterbock M, Rothney E, Harton E, et al. Physical and mental health status of Iraqi refugees resettled in the United States. J Immigr Minor Health. 2013; Epub ahead of print. doi: 10.1007/s10903-013-9893-6

3. Jamil H, Farrag M, Hakim-Larson J, Kafaji T, Abdulkhaleq H, Hammad A. Mental health symptoms in Iraqi refugees: posttraumatic stress disorder, anxiety, and depression. J Culture Diversity. 2007; 14:19-25.

4. Jamil H, Hakim-Larson J, Farrag M, Kafaji T, Duqum I, Jamil L. A retrospective study of Arab American mental health clients: trauma and Iraqi refugees. Am J Orthopsychiatry. 2002; 72:355-61. [PubMed: 15792047]

5. Rhodes C, Chang Y, Percac-Lima S. Development of Obesity and Related Diseases in African Refugees After Resettlement to United States. J Immigr Minor Health. 2015 Epub Sept 21.

6. Sastre L, Haldeman L. Environmental, Nutrition and Health Issues in a US Refugee Resettlement Community. MEDICC Rev. 2015; 17(4):18-24.

7. Dookeran N, Battaglia T, Cochran J, Geltman P. Chronic disease and its risk factgors among refugees and asylees in Massachusetts, 2001-2005. Prev Chronic Dis. 2010; 7(3)

8. Jen K-L, Zhou K, Arnetz B, Jamil H. Pre- and post-displacement stressors and body weight development in Iraqi refugees in Michigan. J Immigr Minority Health. 2015; 17(5):1468-75.

9. Uribe Guajardo M, Slewa-Younan S, Smith M, Eagar S, Stone G. Psychological distress is influenced by length of stay in resettled Iraqi refugees in Australia. Int J Ment Health Syst. 2016; 10(4)doi: 10.1186/s13033-016-0036-Z

10. Jamil H, Aldhalimi A, Arnetz B. Employment Satisfaction and Health Outcomes among Professional Iraqi refugees as compared to Immigrants. J Immigr Refug Stud. 2012; 10(4):395406. [PubMed: 24683383]

11. Appelhans B, Milliron B-J, Woolf K, Johnson TP, SL, Schneider K, Whited M, et al. Socioeconomic status, energy cost, and nutrient content of supermarket food purchases. American journal of preventive medicine. 2012; 42(4)doi: 10.1016/j.amepre.2011.12.007

12. Murayama N. Effects of socioeconomic status on nutrition in Asia and future nutrition policy studies. J Nutr Sci Vitaminol. 2015; 61:S66-S8. [PubMed: 26598891]

13. Yun K, Hebrank K, Graber L, Sullivan M-C, Chen I, Gupta J. High Prevalence of Chronic NonCommunicable Conditions Among Adult Refugees: Implications for Practice and Policy. Journal of Community Health. 2012; 37:1110-8. [PubMed: 22382428] 
14. Wolfe W, Sobal J, Olson C, Frongillo EJ. Parity-associated body weight: modification by sociodemographic and behavioral factors. Obesity research. 1997; 5(2):131-41. [PubMed: 9112248]

15. Brown J, Kaye A, Folsom A. Parity related weight change in women. Int J Obes. 1992; 16:627-31.

16. Nelson-Peterman J, Toof R, Liang S, Grigg-Saito D. Long-Term Refugee Health: Health Behaviors and Outcomes of Cambodian Refugee and Immigrant Women. Health Educ Behav. 2015; 42(6): 814-23. DOI: 10.1177/1090198115590779 [PubMed: 26157042]

17. Perez-Escamilla R, Putnik P. The role of acculturation in nutrition, lifestyle, and incidence of type 2 diabetes among Latinos. J Nutr. 2007; 137:860-70. [PubMed: 17374645]

18. Lara M, Gamboa C, Kahramanian M, Morales L, Bautista D. Acculturation and Latino health in the United States: a review of the literature and its sociopolitical context. Annu Rev Public Health. 2005; 26:367-97. [PubMed: 15760294]

19. Delavari M, Sonderlund A, Swinburn B, Mellor D, Renzaho A. Acculturation and obesity among migrant populations in high income countries - a systematic review. BMC Public Health. 2013; 13:458.doi: 10.1186/1471-2458-13-458 [PubMed: 23663279]

20. Torres S, Nowson C. Relationship between stress, eating behavior, and obesity. Nutrition. 2007; 23:887-94. [PubMed: 17869482]

21. Matheson F, Moineddin R, Glazier R. The weight of place: a multilevel analysis of gender, neighborhood material deprivation, and body mass index among Canadian adults. Soc Sci Med. 2008; 66(3):675-90. DOI: 10.1016/j.socscimed.2007.10.008 [PubMed: 18036712]

22. Laitinen J, Ek E, Sovio U. Stress-related eating and drinking behavior and body mass index and predictors of this behavior. Preventive medicine. 2002; 34:29-39. [PubMed: 11749094]

23. Renzaho A, Bilal P, Marks G. Obesity, type 2 diabetes and high blood pressure amongst recently arrived Sudanese Refugees in Queensland, Australia. J Immigrant Minority Health. 2014; 16:8694.

24. Shoeb M, Weinstein H, Mollica R. The Harvard Trauma Questionnaire: Adapting a cross-cultural instrument for measuring torture, trauma, and posttraumatic stress disorder in Iraqi refugees. Int $\mathrm{J}$ Soc Psychiatr. 2007; 53:447-63.

25. Arnetz B, Broadbridge C, Jamil H, Lumley M, Pole N, Barkho E, et al. Specific trauma subtypes improve the predictive validity of the Harvard Trauma Questionnaire in Iraqi refugees. Journal of Immigrant and Minority Health. 2014; 16:1055-61. [PubMed: 24549491]

26. Weathers F, Litz B, Keane T, Palmieri P, Marx B, Schnurr P. PTSD Checklist for DSM-5 (PCL-5).

27. Zigmond A, Snaith R. The hospital anxiety and depression scale. Acta Psychiatr Scand. 1983; 67:361-70. [PubMed: 6880820]

28. Wagnild G, Young H. Development and psychometric evaluation of the Resilience Scale. J Nurs Meas. 1993; 1(2):165-78. [PubMed: 7850498]

29. Mataeen F, Carone M, Al-Saedy H, Nyce S, Ghosn J, Mutuerandu T, et al. Medical conditions among Iraqi refugees in Jordan: data from the United Nations Refugee Assistance Information System. Bull World Health Organ. 2012; 90:444-51. [PubMed: 22690034] 


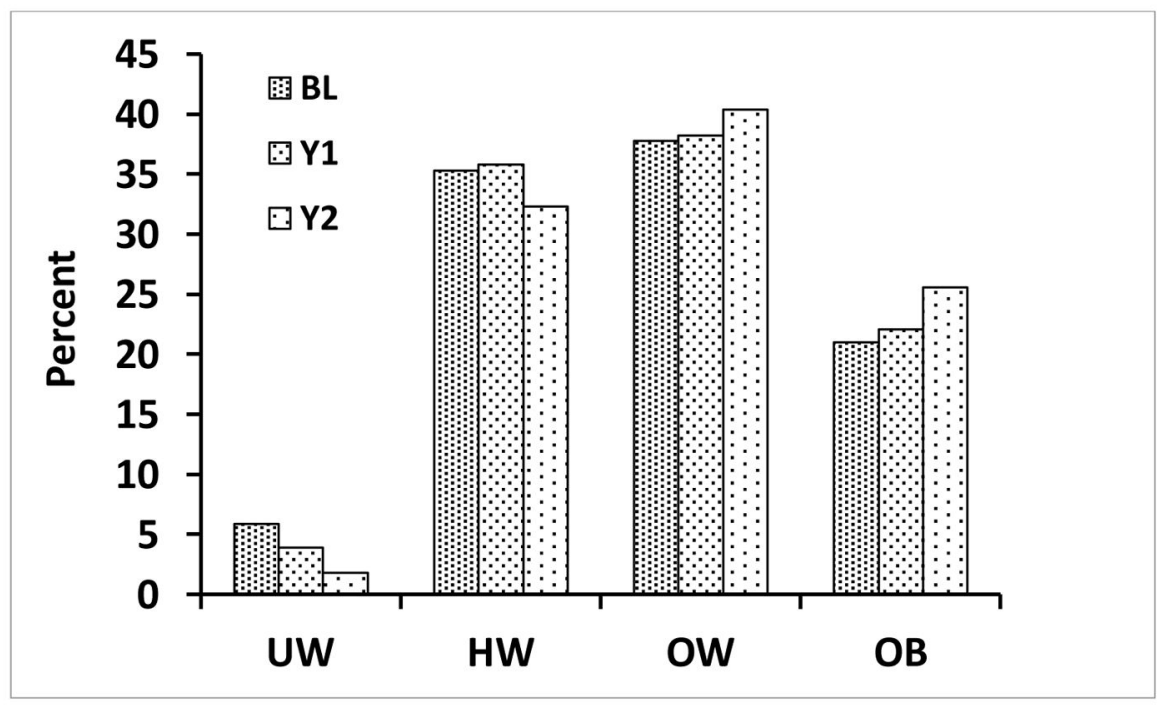

Figure 1.

Percent of refugees classified as underweight, healthy weight, overweight and obese over the 2-year study period. BL: Baseline, Y1: year $1 \mathrm{FU}$; Y2: year $2 \mathrm{FU}$. The upward shift in BMI categories was statistically significant at $\mathrm{p}<.0001$. 


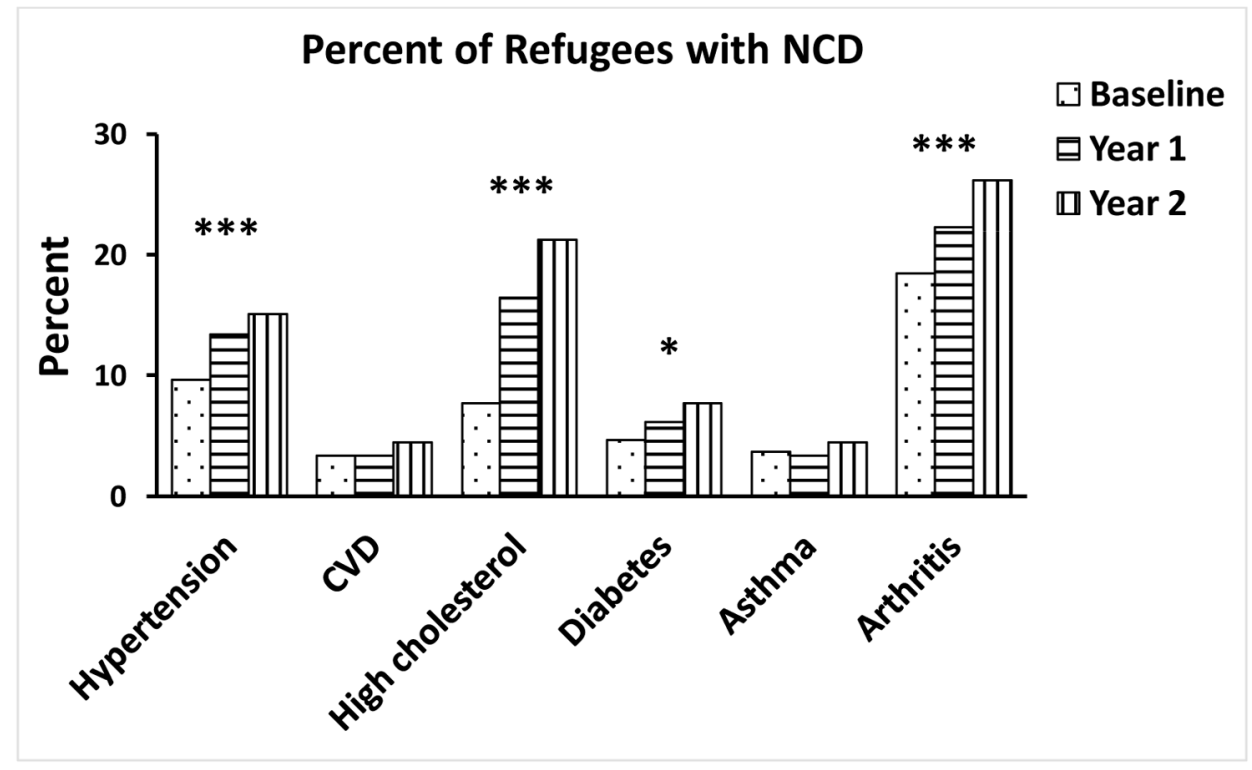

Figure 2.

Percentage of refugees suffering the NCDs at Baseline, year 1 and year 2 .

*: significantly different among the 3 measurement periods at $\mathrm{p}<0.05$

**: significantly different among the 3 measurement periods at $\mathrm{p}<0.01$

***: significantly different among the 3 measurement periods at $\mathrm{p}<0.001$ 


\section{Table 1}

Demographics of the refugees at baseline (BL)

\begin{tabular}{|c|c|}
\hline Sex: & \\
\hline Female: & $137(46 \%)$ \\
\hline Male: & $161(54 \%)$ \\
\hline \multicolumn{2}{|l|}{ Age (years): } \\
\hline & $33.4 \pm 11.3($ mean \pm SD $)$ \\
\hline Range: & $18-69$ \\
\hline \multicolumn{2}{|l|}{ Religion: } \\
\hline Christian: & $269(90.3 \%)$ \\
\hline Muslim: & $25(8.4 \%)$ \\
\hline Mandia: & $4(1.3 \%)$ \\
\hline \multicolumn{2}{|l|}{ Education: } \\
\hline No formal education: & $9(3.0 \%)$ \\
\hline < High school: & $129(43.3 \%)$ \\
\hline High school graduate: & $64(21.5 \%)$ \\
\hline Diploma/certificate: & $50(16.8 \%)$ \\
\hline Bachelor's degree: & $39(13.1 \%)$ \\
\hline Missing data: & $7(2.3 \%)$ \\
\hline \multicolumn{2}{|l|}{ Marital status: } \\
\hline Married: & $154(51.7 \%)$ \\
\hline Single: & $132(44.3 \%)$ \\
\hline Divorced: & $2(0.7 \%)$ \\
\hline Widowed: & $9(3.0 \%)$ \\
\hline Separated: & $1(0.3 \%)$ \\
\hline \multicolumn{2}{|l|}{ Employment (Y2): } \\
\hline Yes & $163(58.2 \%)$ \\
\hline No & $117(41.8 \%)$ \\
\hline
\end{tabular}

J Immigr Minor Health. Author manuscript; available in PMC 2019 April 01. 
Table 2

Measurement instruments administered at BL, Y1 and Y2

\begin{tabular}{ll}
\hline Survey & Instruments \\
Trauma & Harvard Trama Questionnaire [24, 25] \\
PTSD & PTSD Checklist - Civilian Version [26] \\
Depression & Hospital Anxiety and Depression Scale [27] \\
Resilience & Resilience Scale [28] \\
Acculturation & Arab Acculturation Scale [29] \\
\hline
\end{tabular}




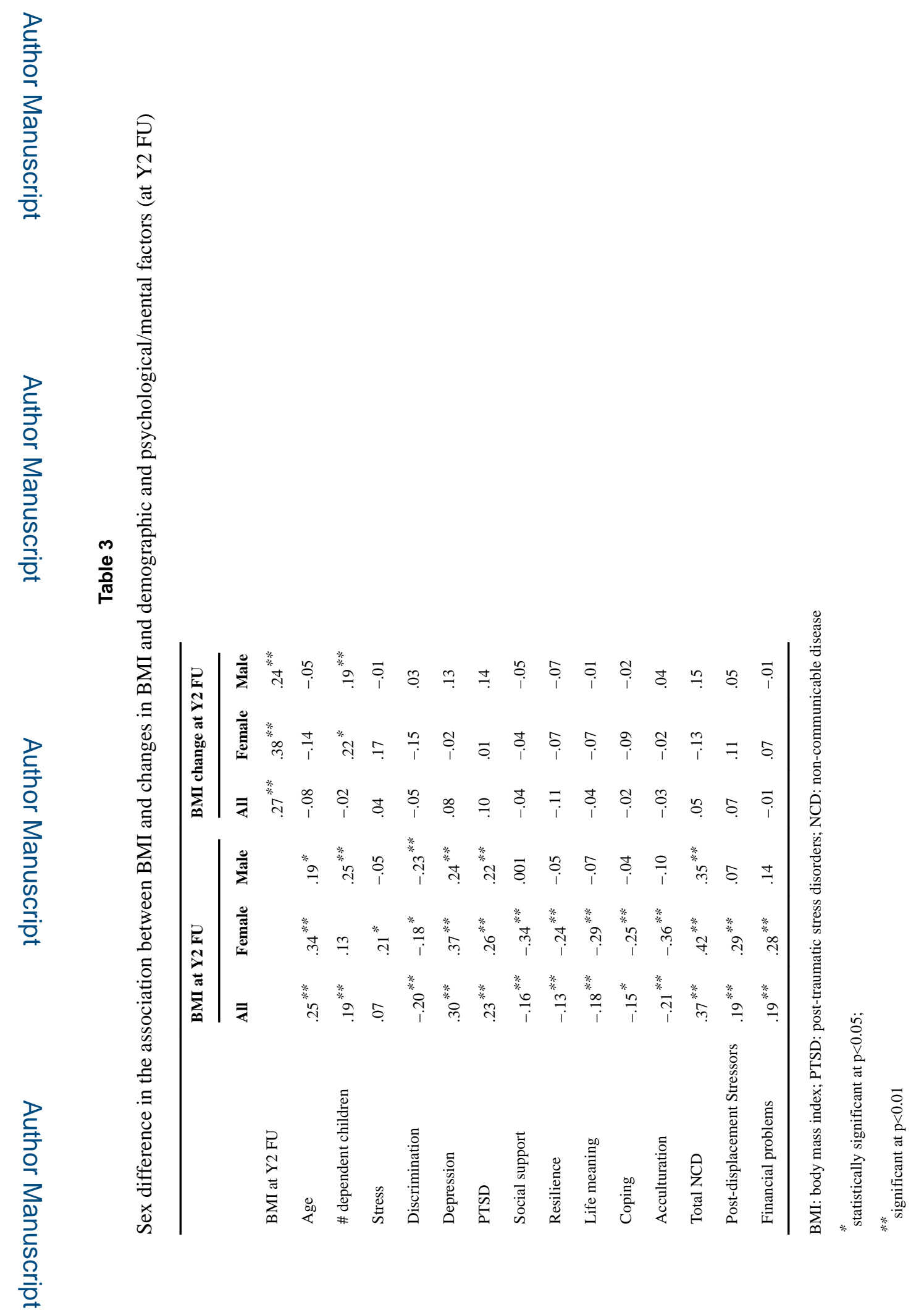

J Immigr Minor Health. Author manuscript; available in PMC 2019 April 01. 
JEN et al.

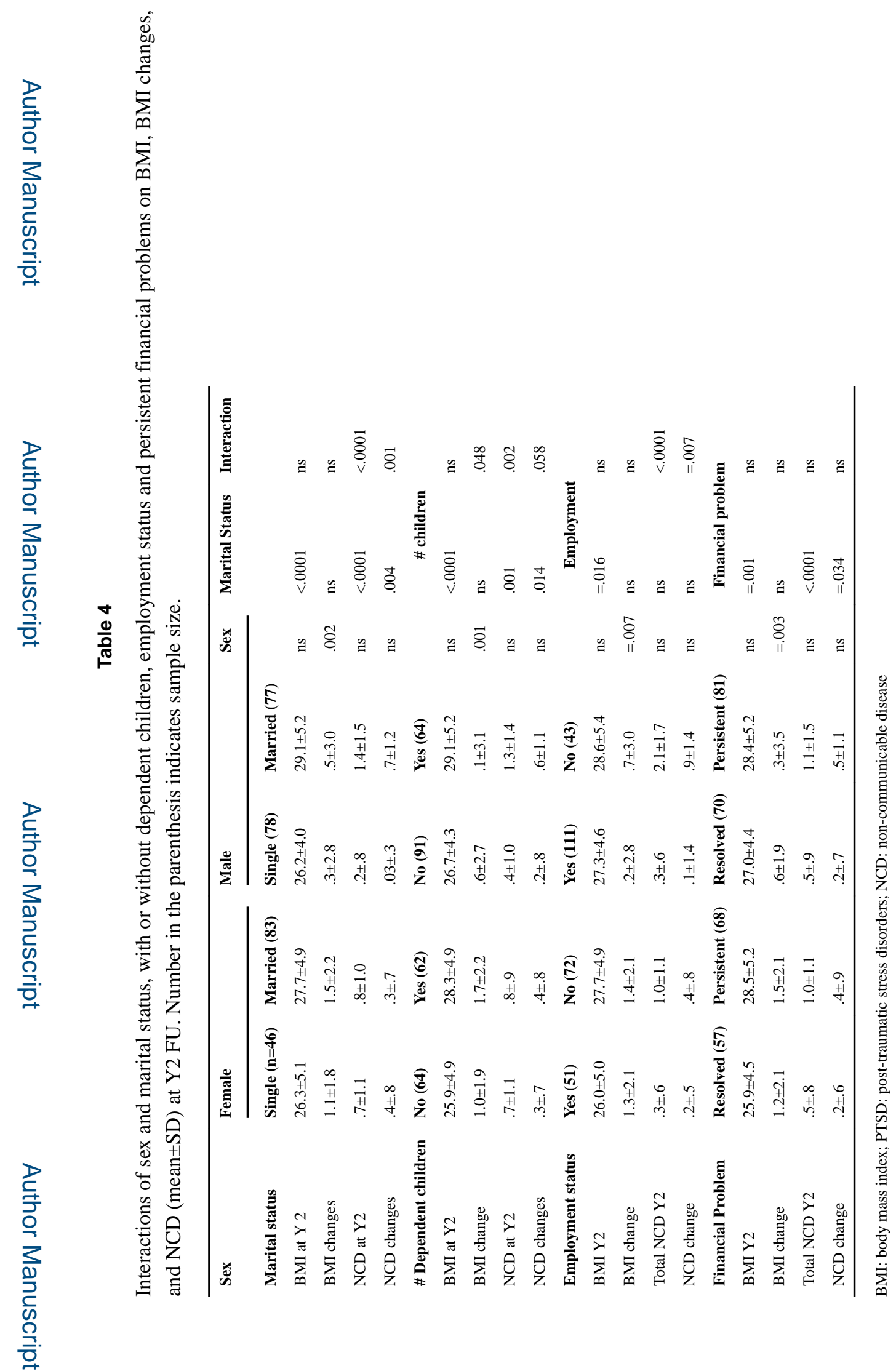

J Immigr Minor Health. Author manuscript; available in PMC 2019 April 01. 


\section{Table 5}

Number of physician-diagnosed NCD (Hypertension, CVD, hypercholesterolemia, diabetes, asthma, and arthritis) in refugees at BL, Y1 and Y2 (number of refugees (\%))

\begin{tabular}{lccc}
\hline \# NCDs & BL & Y1 & Y2 \\
0 & $210(70.5)$ & $188(64.6)$ & $164(57.7)$ \\
1 & $54(18.1)$ & $55(18.9)$ & $60(21.1)$ \\
2 & $19(6.4)$ & $23(7.9)$ & $31(10.9)$ \\
3 & $13(4.4)$ & $16(5.5)$ & $18(6.3)$ \\
4 & $0(0.0)$ & $5(1.7)$ & $7(2.5)$ \\
5 & $1(0.3)$ & $3(1.0)$ & $3(1.1)$ \\
6 & $1(0.3)$ & $1(0.3)$ & $1(0.4)$ \\
\hline
\end{tabular}

NCD: non-communicable disease; CVD: cardiovascular disease; BL: baseline 


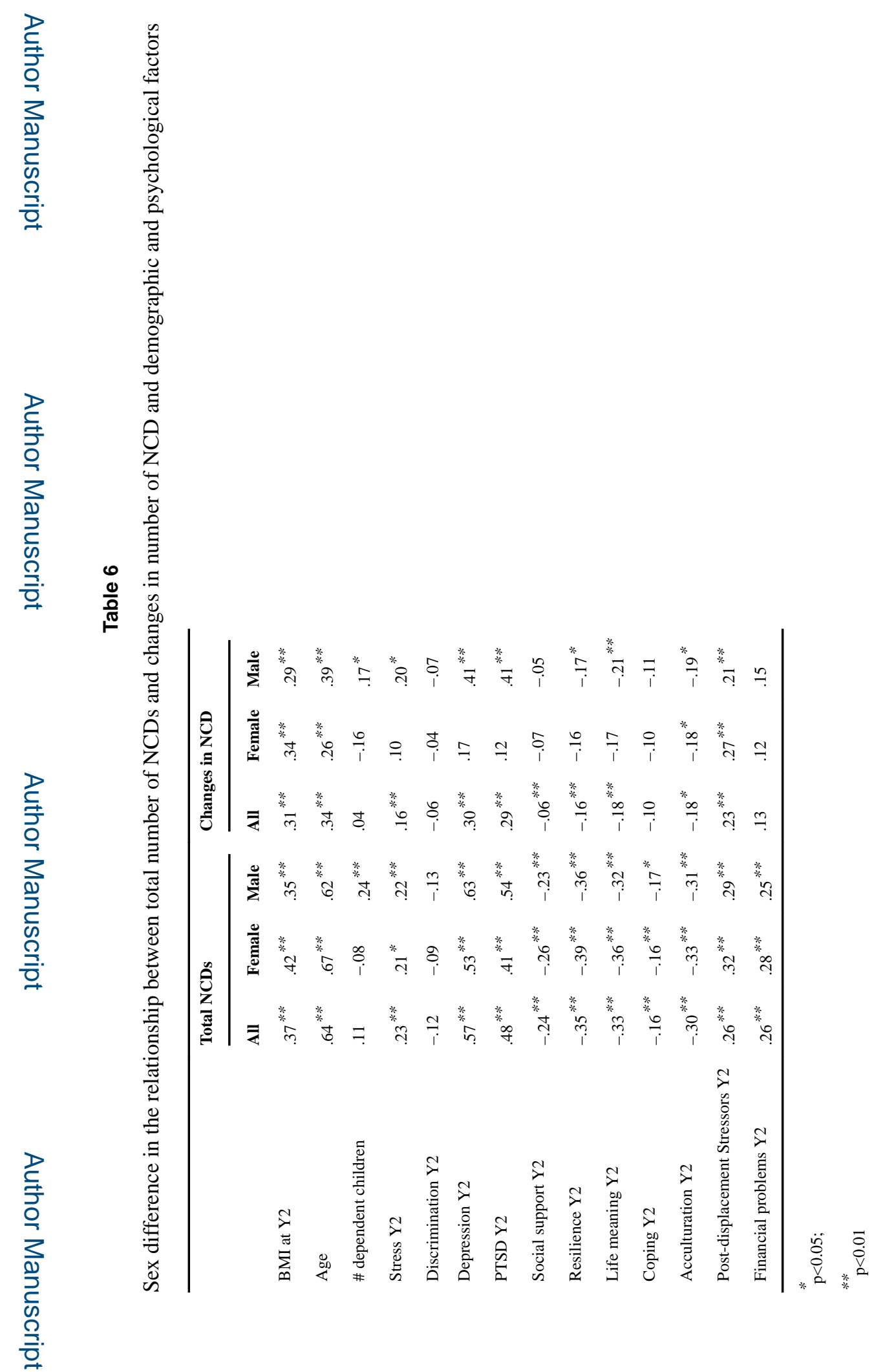

J Immigr Minor Health. Author manuscript; available in PMC 2019 April 01. 


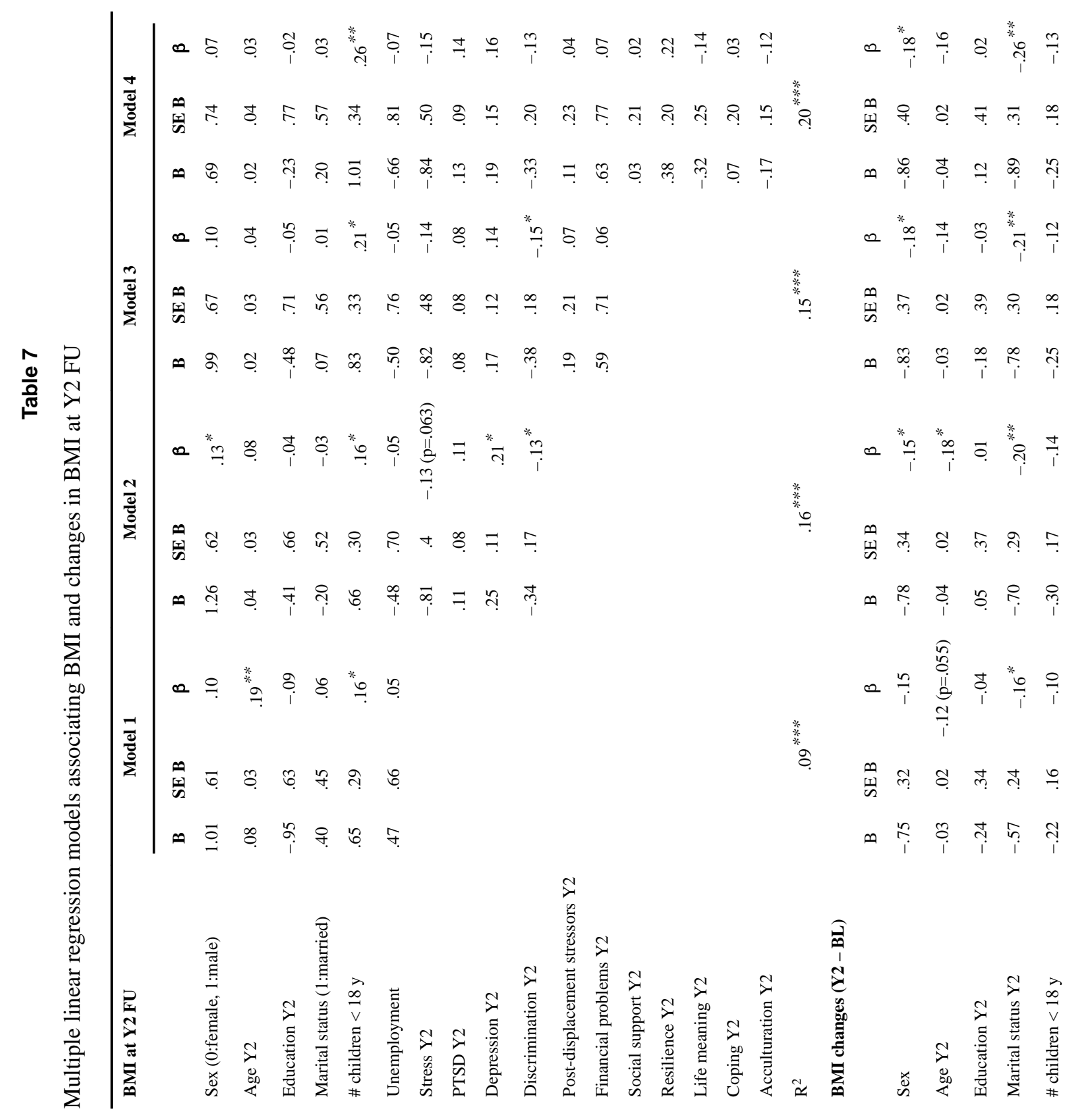

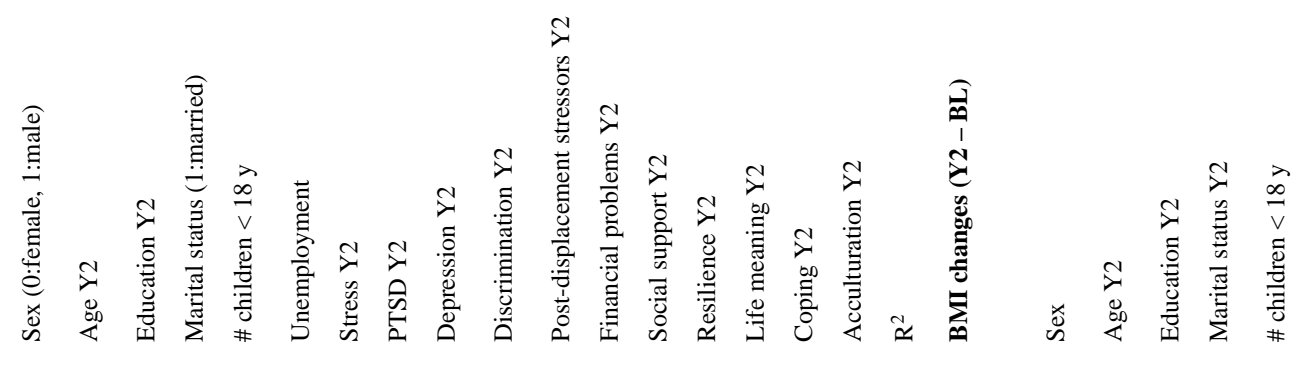

J Immigr Minor Health. Author manuscript; available in PMC 2019 April 01. 
JEN et al.

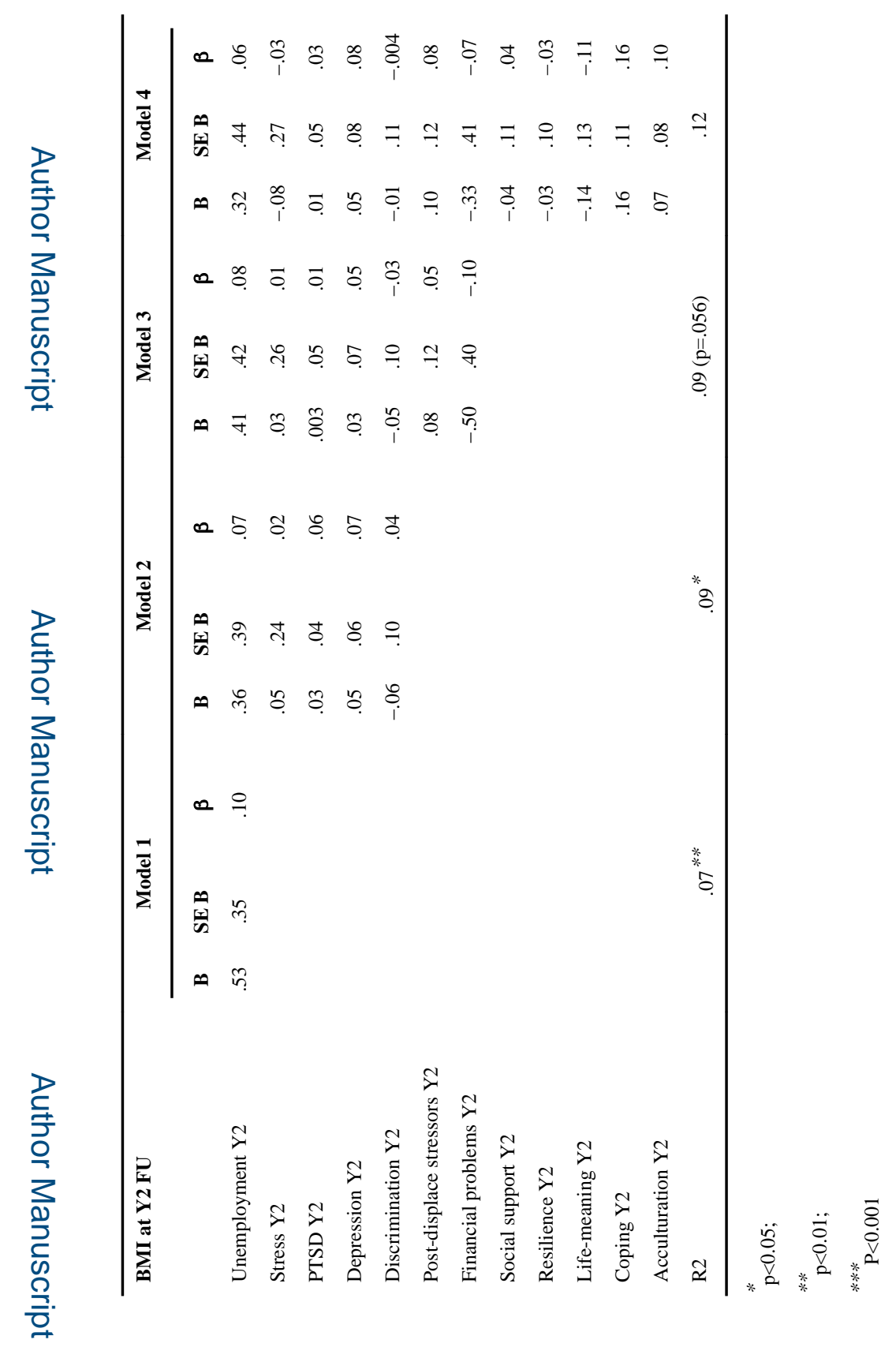

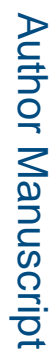

J Immigr Minor Health. Author manuscript; available in PMC 2019 April 01. 


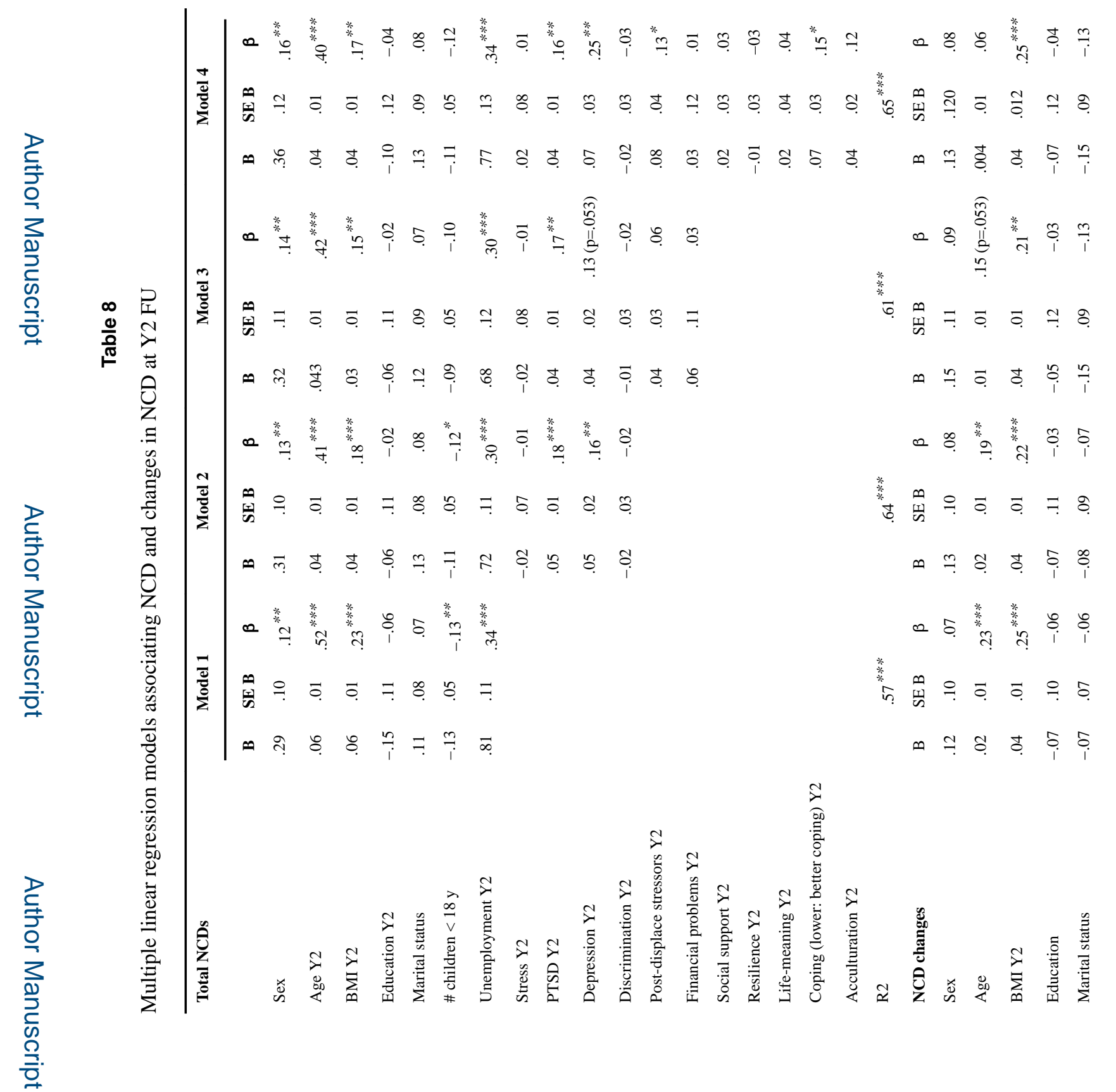

J Immigr Minor Health. Author manuscript; available in PMC 2019 April 01. 
JEN et al.

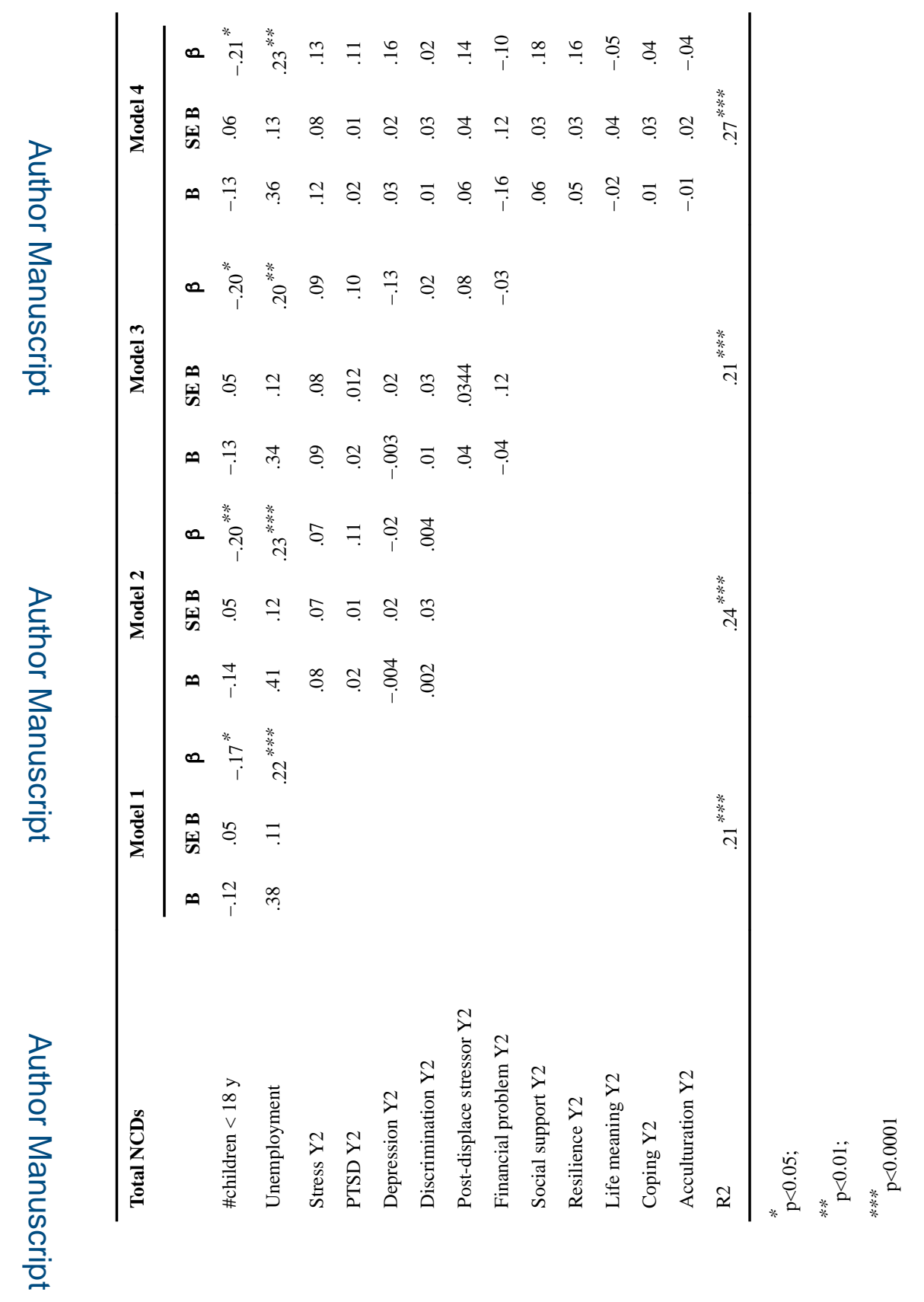

로을

J Immigr Minor Health. Author manuscript; available in PMC 2019 April 01. 